\title{
A MIXED-FRAME-OF-REFERENCE MODEL FOR RAILWAY INDUCED SOIL-STRUCTURE VIBRATION INTERACTION
}

\author{
Paulius Bucinskas $^{1}$, Evangelos Ntotsios ${ }^{2}$, David J. Thompson ${ }^{2}$ and Lars V. Andersen ${ }^{1}$ \\ ${ }^{1}$ Department of Engineering \\ Aarhus University \\ Denmark \\ (pb, lva)@eng.au.dk \\ ${ }^{2}$ Institute of Sound and Vibration Research \\ University of Southampton \\ United Kingdom \\ e.ntotsios@soton.ac.uk,djt@isvr.soton.ac.uk
}

Keywords: Soil-structure interaction, train induced vibration, mixed frame of reference

\begin{abstract}
When modelling the railway induced vibration in a building, three main aspects of the problem have to be considered: the vibration generation at the moving vehicle-track interaction points, the vibration propagation in the underlying infinite soil, and the vibration reception inside the building. This study proposes a computational modelling approach for predicting the full vibration propagation path from the train vehicles up to the building structure. The method includes a moving train model that is directly coupled to a stationary building structure, with interaction between them through the underlying soil using a single step solution procedure. A semi-analytical model is utilized to model the soil to which rigid objects and structures modelled by finite elements (FE) are coupled. The system is excited by a multi-body vehicle model passing over an irregular track. The proposed modelling approach uses the frequency-domain solution with some parts, such as the railway track, formulated in the moving frame of reference (FOR) and other parts, such as building structures, formulated in a fixed FOR. The coupling terms between the two FORs are found by utilizing an analytical formulation of receptance between the two FORs. It is shown that due to the coupling between the fixed and moving FORs, the previously uncoupled discrete frequencies become coupled through the other FOR as a result of the Doppler effect and wave scattering. Two solution procedures of the full system are proposed: partial coupling, where some secondary effects from reflected waves propagating through soil are disregarded, and full coupling, where the vehicle, track, soil and structure are modelled as a fully coupled system. Both proposed solution procedures offer a single-step approach for solving the whole system in the frequency-spatial domain. The application of the model is demonstrated and validated in two example cases: one analyzing a simple building structure near a railway track, using the partial coupling solution procedure, and another analyzing the behaviour of a vehicle model passing over a rigid block embedded inside the soil, using the full coupling solution procedure.
\end{abstract}




\section{INTRODUCTION}

One of the important environmental impacts of existing and future railway lines occurs due to the ground-borne vibration induced by the trains. When a train runs on the track, its wheels induce vibration in the track structure. This vibration then propagates through the ground into nearby buildings where it is perceived either directly, due to motion of floors and walls, or indirectly as re-radiated noise. This causes disturbance to occupants, potential loss of building functionality (e.g. interference with sensitive equipment), loss of real estate value and, in worst case, leading to structural damage.

The impact of railway-induced vibrations has been investigated since the beginning of the previous century and much work has been done to understand and simulate the dynamic interactions between the train, track, tunnel (for underground railways) and ground resulting in models which can predict ground-borne vibration around the railway system. The exact propagation mechanism of environmental vibration is an extremely complex phenomenon, and thus proper evaluation of its effects is difficult. Several different numerical models have been developed for predicting vibration from surface and underground railways in the last few decades. They are mainly based on numerical or semi-analytical methods. Each approach has its own merits. Semi-analytical models are simpler, requiring less input parameters, but they are not able to represent the full details of a given situation. Conversely, numerical models can represent more detailed geometry but require a larger number of input parameters and greater computational effort. The results are then specific to this particular situation and cannot be generalized easily. There are also important limitations in the use of either type of model, due to the modelling simplifications and parameter uncertainty. A comprehensive overview of the state of the art on railway-induced ground vibration models and the underlying excitation mechanisms can be found in [1].

When modelling the environmental vibration in a building induced by railway traffic, three main parts of the problem need to be considered: the vehicle-track system which is the vibration source, the underlying soil through which the vibration propagates, and the building structure which is the vibration receiver. Ideally, all the parts would be combined into a single model, including all coupling terms. However, due to complex analytical formulation needed and the limitations of computational models, this is rarely performed. Most often, parts of the model are coupled together; for example, a coupled vehicle-track-soil model is calculated, and the obtained results propagate to the building structure [2 - 9]. This way, the secondary coupling terms are excluded from the system, such as the effect of the building structure to the response of the track and the vehicle. This is acceptable, as these coupling terms do not influence the system significantly [10]. For example, Fiala et al. [2] used a two-step approach to obtain the response of a building structure by splitting the problem into a vehicle-track-soil source model and a structure-soil receiver model. With a similar approach, François et al. [3] studied roadtraffic-induced vibration. The work also proposed a methodology to exclude the soil-structure interaction problem for cases of soft structures resting on stiff soils. A so-called sub-modelling technique was proposed by Hussein et al. [6], where the response of the soil surface from a tunnel structure was modelled using the 'pipe-in-pipe' model [11] to find the response of a 2D frame. An almost identical system assembly method was also used by Lopes et al. [5]; however, the soil surface response from a tunnel structure was modelled using a 2.5D FEM-PML (perfectly matched layers) model and a 3D building structure was analyzed. In [9], Kuo et al. use a similar numerical approach and the model developed in [10] to investigate the effect of dynamic soil characteristics, surface foundation type, and building geometry on the building's response to railway induced vibrations and compare with the FRA empirical adjustment factors [12]. 
The aim of this work is to model the full vibration propagation path from the vehicle up to the building structure. The proposed method includes a moving vehicle model that is directly coupled to a stationary building structure, with interaction between them through the infinite underlying soil, without needing a multiple step solution. At the same time, the method offers a relatively quick and flexible solution procedure. A fully 3D system is modelled, with structures modelled using FE, allowing a wide variety of configurations. To limit the computational times needed, the semi-analytical soil formulation is utilized, with a surface railway track, using the model originally proposed by Sheng et al. in [13, 14] and later extended in [15]. A multibody vehicle model passing over an irregular track excites the system. The work introduces an approach of solving the coupled vehicle-track-soil-building system using a single step solution procedure. Two system assembly and solution methods are presented: partial coupling and full coupling. With the partial coupling procedure disregarding some effects caused by the reflected waves for a more computationally efficient solution, and the full coupling procedure including all the coupling terms. Additionally, the methodology for modelling rigid objects and FE structures interacting with the soil is described with its implementation into the proposed solution approaches. To show the capabilities of the proposed modelling approach two example cases are analyzed: (i) for a simple building structure near a railway track, using the partial coupling solution procedure, and (ii) the behaviour of a vehicle model passing over a rigid block embedded inside the soil, using the full coupling solution procedure.

\section{SEMI-ANALYTICAL SOIL MODEL}

A semi-analytical soil model is used in the present work. The model utilizes a well-known approach based on an analytical solution to the Green's function in the frequency-wavenumber domain. For the linear hysteric half-space $z \leq 0$, the displacement field in time-space domain can be obtained using a convolution integral:

$$
u_{i}(x, y, z, t)=\int_{-\infty}^{t} \int_{-\infty}^{0} \int_{-\infty}^{\infty} \int_{-\infty}^{\infty} g_{i j}\left(x-x^{\prime}, y-y^{\prime}, z, z^{\prime}, t-t^{\prime}\right) p_{j}\left(x^{\prime}, y^{\prime}, z^{\prime}, t^{\prime}\right) \mathrm{d} x^{\prime} \mathrm{d} y^{\prime} \mathrm{d} z^{\prime} \mathrm{d} t^{\prime}
$$

where the Green's function $g_{i j}$ relates the displacement component $u_{i}$ at the point $(x, y, z)$ and time $t$ to the loads $p_{j}$ applied in direction $j$ at all positions and times up to and including the time $t$. The soil is assumed invariant and infinite in both horizontal directions, while the material properties vary over depth due to stratification.

The Green's function is challenging to find analytically in time-space domain for a layered half-space. Thus, a triple Fourier transformation can be performed, transforming the two horizontal coordinates into the wavenumbers $k_{x}$ and $k_{y}$, and time into the circular frequency $\omega$. By introducing a discretization into a number of depths $z_{n}, n=1,2,3, \ldots, N_{z}$, Eq. (1) simplifies into

$$
\bar{U}_{i}\left(k_{x}, k_{y}, z, \omega\right)=\sum_{n=1}^{N_{z}} \bar{G}_{i j}\left(k_{x}, k_{y}, z, z_{n}, \omega\right) \bar{P}_{n, j}\left(k_{x}, k_{y}, \omega\right)
$$

where $\bar{U}$ and $\bar{G}_{i j}$ are components of the displacement vector and the Green's function tensor, respectively, in the frequency-wavenumber domain. After the discretization over depth, $\bar{P}_{n, j}$ signifies the traction applied on a horizontal interface placed at the depth $z_{n}$. In the following, upper case symbols indicate Fourier transforms with respect to time, whereas overbar indicates Fourier transforms with respect to the horizontal spatial coordinates.

There are two major approaches used to assemble multiple soil layers and express analytically the Green's functions $\bar{G}_{i j}$ appearing in Eq. (2): the flexibility approach and the stiffness 
approach. The flexibility approach is based on the original work proposed by Thomson [16] and Haskell [17]. The stiffness approach was introduced by Kausel and Roësset [18] and extends the original transfer matrix, as derived in $[16,17]$ by reordering it into a stiffness expression equivalent to those used in the FEM, assuming that the interface between two layers is interpreted as a connecting node. Multiple layers can be assembled by overlapping the stiffness matrices at the connecting interfaces.

In this work, both methods have been utilized: the flexibility approach with numerical stabilization for the fixed FOR soil model and the stiffness approach for the moving FOR model. The reason that the flexibility approach is used for the fixed FOR and the soil-structure interaction is because it enables the application of structure-to-soil interaction forces without increasing the size of the involved matrices (that is always six by six) for foundations that are embedded in the ground (i.e. piled foundations). For the moving FOR problem, only the Green's functions for a surface load (i.e. the track) are needed and thus the stiffness approach is used which is free of numerical instabilities at higher frequencies, that are more prominent due to the moving of the load. Any instabilities occurring in the flexibility approach are dealt using stabilization techniques that are based on the orthonormalization method [19] and by splitting very thick layers (if any) into multiple smaller layers with the same material properties.

After the displacements in the frequency-wavenumber domain are obtained, a double inverse Fourier transformation is performed into frequency-space domain

$$
U_{\mathrm{f} i}\left(x_{\mathrm{f}}, y, z, \omega_{\mathrm{f}}\right)=\frac{1}{4 \pi^{2}} \int_{-\infty}^{\infty} \int_{-\infty}^{\infty} \bar{U}_{i}\left(k_{x}, k_{y}, z, \omega_{\mathrm{f}}\right) \mathrm{e}^{\mathrm{i}\left(k_{x} x_{\mathrm{f}}+k_{y} y\right)} \mathrm{d} k_{x} \mathrm{~d} k_{y} .
$$

This equation is used when both the load and the response in a fixed FOR is considered. The load is applied at a circular frequency $\omega_{\mathrm{f}}$ and coordinate $x_{\mathrm{f}}$, with subscript ' $\mathrm{f}$ ' indicating a fixed FOR. Here, the inverse Fourier transformation can be carried out in semi-discrete form by adopting polar coordinates, since the integration with respect to the azimuthal angle can be done in closed form, leading to Bessel functions in the components of the Green's function.

When the whole system is considered in a moving FOR, the Green's function and, at the same time, the response in the frequency-wavenumber domain lose the polar symmetry around the origin of the wavenumber domain, compared to a purely fixed FOR. Hence, a fully discrete inverse Fourier transformation from wavenumber domain into spatial domain is necessary. Assuming that a load is moving in the positive x-direction, the frequency used to compute the Green's function becomes wavenumber dependent. In that case, the inverse double Fourier transformation into frequency-space domain is defined as

$$
U_{\mathrm{m} i}\left(x_{\mathrm{m}}, y, z, \omega_{\mathrm{m}}\right)=\frac{1}{4 \pi^{2}} \int_{-\infty}^{\infty} \int_{-\infty}^{\infty} \bar{U}_{i}\left(k_{x}, k_{y}, z, \omega_{\mathrm{m}}-k_{x} v\right) \mathrm{e}^{\mathrm{i}\left(k_{x} x_{\mathrm{m}}+k_{y} y\right)} \mathrm{d} k_{x} \mathrm{~d} k_{y}
$$

where $v$ is the velocity at which the moving FOR travels through the fixed FOR. Subscript ' $m$ ' indicates values in the moving FOR.

Since the methodology presented in this work uses both moving and fixed FOR, a mixed FOR is used, where the displacement response observed in a fixed FOR from a load applied at a stationary point in the moving FOR and vice versa are needed in order to obtain the coupling terms between the two FORs.

Following a similar analysis with [13], the displacements $U_{\mathrm{f} i}$ at $x_{\mathrm{f}}$ for fixed FOR from a harmonic moving load of circular frequency $\omega_{\mathrm{m}}$ can be expressed as

$$
U_{\mathrm{f} i}\left(x_{\mathrm{f}}, y, z, \omega_{\mathrm{m}}, \omega_{\mathrm{f}}\right)=\frac{1}{v} \mathrm{e}^{\mathrm{i} \beta x_{\mathrm{f}}} \frac{1}{2 \pi} \int_{-\infty}^{\infty} U_{i}\left(\beta, k_{y}, z, \omega_{\mathrm{m}}-\beta v\right) \mathrm{e}^{\mathrm{i} k_{y} y} \mathrm{~d} k_{y}, \quad \beta=\frac{\omega_{\mathrm{m}}-\omega_{\mathrm{f}}}{v}
$$


where $\omega_{\mathrm{f}}$ is the receiving frequency at the fixed FOR.

Alternatively, using an equivalent analysis for a stationary harmonic load of circular frequency $\omega_{m}$ received in a moving FOR at $x_{\mathrm{m}}=x_{\mathrm{f}}-v t$ the displacements $U_{\mathrm{m} i}$ can be expressed as:

$$
U_{\mathrm{m} i}\left(x_{\mathrm{m}}, y, z, \omega_{\mathrm{f}}, \omega_{\mathrm{m}}\right)=\frac{1}{v} \mathrm{e}^{\mathrm{i} \beta x_{\mathrm{m}}} \frac{1}{2 \pi} \int_{-\infty}^{\infty} U_{i}\left(\beta, k_{y}, z, \omega_{\mathrm{f}}\right) \mathrm{e}^{\mathrm{i} k_{y} y} \mathrm{~d} k_{y}, \quad \beta=\frac{\omega_{\mathrm{m}}-\omega_{\mathrm{f}}}{v} .
$$

Comparing Eqs. (5) and (6) it is evident that the expressions are equivalent. That is, the displacements originating from a moving source and observed in a fixed FOR are equivalent to the displacements caused by a stationary source and observed in a moving frame:

$$
U_{\mathrm{m} i}\left(x_{\mathrm{m}}, y, z, \omega_{\mathrm{f}}, \omega_{\mathrm{m}}\right)=U_{\mathrm{f} i}\left(x_{\mathrm{f}}, y, z, \omega_{\mathrm{m}}, \omega_{\mathrm{f}}\right)
$$

given that the horizontal coordinates $x_{\mathrm{m}}$ and $x_{\mathrm{f}}$ have the same numerical value. In practice, this means that only Eq. (5) or Eq. (6) needs to be evaluated. Then the integral part of the equation is reused for the other FOR combination, with changed $x$-coordinate.

\section{STRUCTURES INTERACTING WITH SOIL IN A SINGLE FRAME OF REFERENCE}

To couple the semi-analytical soil model to a FE model of one or more structures, a dynamic stiffness matrix of the soil is established. Firstly, the desired geometry of the soil-structure interface is discretized into a number of 'soil-structure interaction' (SSI) nodes. The response is requested at a range of 'observation' nodes. The dynamic stiffness matrix is established using the SSI nodes, which is then coupled to structures and used to obtain the system displacements.

To establish the dynamic stiffness matrix of the soil, a global flexibility matrix relating all the degrees of freedom is needed. For a three-dimensional case, each SSI node has three degrees of freedom. Therefore, the receptance matrix $\mathbf{R}_{\mathrm{ss}}(\omega)$, also called the flexibility matrix, is a square matrix with three times more rows and columns than the number of nodes in the system. To create the receptance matrix, a unit harmonic load $\bar{P}_{0, s_{j}}$ is applied to a single degree of freedom, $s_{j}$, and the resulting displacements are observed at all SSI degrees of freedom, including the loaded one. The procedure is repeated for every degree of freedom in the system. This way, the receptance relating all degrees of freedom to the loaded degree of freedom is established. The receptance between degrees of freedom $s_{i}$ and $s_{j}$ is found as

$$
R_{s_{i}, S_{j}}(\omega)=\int_{-\infty}^{\infty} \int_{-\infty}^{\infty} \bar{G}_{d_{i} d_{j}}\left(k_{x}, k_{y}, z_{n_{i}}, z_{n_{j}}, \omega\right) \hat{\bar{P}}_{n_{j}, S_{j}}\left(k_{x}, k_{y}, \omega\right) \mathrm{e}^{\mathrm{i}\left(k_{x}\left(x_{n_{i}}-x_{n_{j}}\right)+k_{y}\left(y_{n_{i}}-y_{n_{j}}\right)\right)} \mathrm{d} k_{x} \mathrm{~d} k
$$

where $\hat{\bar{P}}_{n_{j}, s_{j}}\left(k_{x}, k_{y}, \omega\right)$ is the double spatial Fourier transform of a distributed harmonic load $\hat{P}_{n_{j}, s_{j}}(x, y, \omega)$ of unit magnitude acting within the frequency domain at the depth $z_{n_{j}}$ in degree of freedom $s_{j}$. The same procedure can be performed in both fixed and moving FORs. The obtained results are placed in a single column of the receptance matrix and the process is repeated for every degree of freedom. Assuming there is a total of $S$ SSI degrees of freedom in the system, the assembled receptance matrix becomes:

$$
\mathbf{R}_{\mathrm{sS}}(\omega)=\left[\begin{array}{cccc}
R_{s_{1}, s_{1}}(\omega) & R_{s_{1}, s_{2}}(\omega) & \cdots & R_{s_{1}, s_{S}}(\omega) \\
R_{S_{2}, s_{1}}(\omega) & R_{S_{2}, s_{2}}(\omega) & \cdots & R_{s_{2}, s_{S}}(\omega) \\
\vdots & \vdots & \ddots & \vdots \\
R_{s_{S}, s_{1}}(\omega) & R_{S_{S}, s_{2}}(\omega) & \cdots & R_{S_{S}, s_{S}}(\omega)
\end{array}\right] .
$$


The receptance matrix can be established for the moving and fixed FORs. However, only in the fixed FOR, the matrix is symmetric, assuming that the applied load $\hat{P}_{n_{j}, s_{j}}(x, y, \omega)$ is the same for all degrees of freedom. If no external structures are connected to the semi-analytical soil model, it is possible to apply the loads and obtain the system displacements $\mathbf{U}_{\mathrm{s}}$ directly as

$$
\mathbf{U}_{\mathrm{s}}(\omega)=\mathbf{R}_{\mathrm{sS}}(\omega) \mathbf{P}_{\mathrm{S}}(\omega)
$$

where $\mathbf{P}_{\mathbf{S}}$ is the vector of load magnitudes. It is implied here that the load acting in degree of freedom $s_{j}$ has a spatial distribution defined by $\widehat{P}_{n_{j}, s_{j}}$ and a magnitude defined by element $j$ of $\mathbf{P}_{\mathrm{s}}$.

\subsection{FE models interacting with the soil}

To couple the soil model with an FE model of one or more structures, the dynamic stiffness matrix of the soil $\mathbf{K}_{\mathrm{ss}}(\omega)$ is needed. It can be obtained by inverting the receptance matrix. Including the dynamic stiffness matrix $\mathbf{K}_{\mathrm{FE}}$ of the FE model the dynamic stiffness in the single frame of reference (SFOR) can be assembled as:

$$
\mathbf{K}_{\mathrm{SFOR}}(\omega)=\left[\begin{array}{cc}
\mathbf{K}_{\mathrm{ss}}(\omega)+\mathbf{K}_{\mathrm{FE}}^{\mathrm{ss}}(\omega) & \mathbf{K}_{\mathrm{FE}}^{\mathrm{sn}}(\omega) \\
\mathbf{K}_{\mathrm{FE}}^{\mathrm{ns}}(\omega) & \mathbf{K}_{\mathrm{FE}}^{\mathrm{nn}}(\omega)
\end{array}\right]
$$

where superscript ' $\mathrm{s}$ ' denotes the degrees of freedom through which the FE model interacts with the soil, while superscript ' $n$ ' denotes the degrees of freedom that are internal to the FE model. Combinations of superscripts 'sn' and 'ns' denote the coupling terms. Due to symmetry of the FE system matrices, $\mathbf{K}_{\mathrm{FE}}^{\mathrm{ns}}(\omega)=\left[\mathbf{K}_{\mathrm{FE}}^{\mathrm{sn}}(\omega)\right]^{\mathrm{T}}$. In addition, the matrix $\mathbf{K}_{\mathrm{FE}}^{\mathrm{sn}}(\omega)$ is usually sparsely populated.

The displacements of the system can then be obtained by solving the system of equations:

$$
\mathbf{K}_{\mathrm{SFOR}}(\omega) \mathbf{U}_{\mathrm{SFOR}}(\omega)=\mathbf{P}_{\mathrm{SFOR}}(\omega) \text {. }
$$

To obtain the displacements of the observation degrees of freedom, a flexibility matrix relating the SSI and observation degrees of freedom is needed. Assuming that the numbering of observation degrees of freedom is stored in a set $o=\left\{o_{1}, o_{2}, \ldots, o_{O}\right\}$ with the number of degrees of freedom being $O$, the flexibility matrix will have $O$ rows and $S$ columns. The flexibility matrix for observation degrees of freedom is assembled as:

$$
\mathbf{R}_{\mathrm{os}}(\omega)=\left[\begin{array}{cccc}
R_{o_{1}, s_{1}}(\omega) & R_{o_{1}, s_{2}}(\omega) & \cdots & R_{o_{1}, s_{S}}(\omega) \\
R_{O_{2}, s_{1}}(\omega) & R_{o_{2}, s_{2}}(\omega) & \cdots & R_{o_{2}, s_{S}}(\omega) \\
\vdots & \vdots & \ddots & \vdots \\
R_{o_{O}, s_{1}}(\omega) & R_{o_{O}, s_{2}}(\omega) & \cdots & R_{o_{O}, s_{S}}(\omega)
\end{array}\right] .
$$

To find the displacements of the observation nodes, the observation flexibility matrix $\mathbf{R}_{\mathrm{os}}$ is multiplied by the soil displacements at the SSI degrees of freedom $\mathbf{U}_{\mathbf{s}}$, which are extracted from the whole system displacement vector $\mathbf{U}_{\mathrm{SFOR}}$ :

$$
\mathbf{U}_{\mathrm{o}}(\omega)=\mathbf{R}_{\mathrm{os}}(\omega)\left[\mathbf{R}_{\mathrm{ss}}(\omega)\right]^{-1} \mathbf{U}_{\mathrm{s}}(\omega)=\mathbf{R}_{\mathrm{os}}(\omega) \mathbf{K}_{\mathrm{ss}}(\omega) \mathbf{U}_{\mathrm{s}}(\omega) .
$$

\subsection{Rigid structures interacting with the soil}

The semi-analytical soil model allows also modelling of completely rigid objects interacting with the soil. This is useful when modelling structures that are much stiffer than the surrounding material, for example building foundations. To create a three-dimensional rigid object, the object shape is discretized into a number of SSI nodes. The global flexibility matrix is created in 
the same way as in the previous subsection and inverted to obtain the dynamic stiffness matrix. However, condensation of the stiffness matrix must be performed in order to reduce the system such that the response can be determined in terms of the rigid body modes rather than the original degrees of freedom of the SSI nodes. This is achieved by assuming that SSI nodes belonging to the same rigid object are fixed relatively to each other and move together with the degrees of freedom of a reference master node that defines the motion of the rigid object. In principle, the master node can be placed at any position. However, it is most conveniently placed in the point at which coupling to an FE model should be done. That is, for example, in the centre of the topside of a footing. In the most common three-dimensional case, separate SSI nodes have three degrees of freedom each, i.e. three lateral displacements, while each rigid object has six degrees of freedom: three for lateral displacements and three additional rotational degrees of freedom of the reference node. For a single rigid object composed of a number of SSI nodes, the transformation matrix $\mathbf{T}_{0, i}$ is created. Multiple rigid objects can also be a part of the same system. The global transformation matrix for a system with $N$ rigid objects can be assembled as:

$$
\mathbf{T}_{\mathrm{S}}=\left[\begin{array}{cccc}
\mathbf{T}_{0,1} & \mathbf{0} & \cdots & \mathbf{0} \\
\mathbf{0} & \mathbf{T}_{0,2} & \cdots & \mathbf{0} \\
\vdots & \vdots & \ddots & \vdots \\
\mathbf{0} & \mathbf{0} & \cdots & \mathbf{T}_{0, N}
\end{array}\right]
$$

The system can also contain non-associated 'free' SSI nodes, which are not part of any rigid object. In that case, the local transformation matrix for such nodes will be the identity matrix with the same number of rows and columns as the number of degrees of freedom associated with the free node.

To obtain the condensed stiffness matrix of the soil, , the dynamic stiffness matrix of the soil $\mathbf{K}_{\mathrm{ss}}(\omega)$ is modified:

$$
\mathbf{K}_{\mathrm{s}}(\omega)=\left[\mathbf{T}_{\mathrm{s}}\right]^{\mathrm{T}} \mathbf{K}_{\mathrm{ss}}(\omega) \mathbf{T}_{\mathrm{s}} .
$$

The matrix can then be coupled to FE structures in the same way as described previously. Due to condensation of some SSI nodes, Eq. (14) is also modified by introducing the transformation matrix

$$
\mathbf{U}_{\mathrm{o}}(\omega)=\mathbf{R}_{\mathrm{os}}(\omega)\left[\mathbf{R}_{\mathrm{ss}}(\omega)\right]^{-1} \mathbf{T}_{\mathrm{s}} \mathbf{U}_{\mathrm{s}}(\omega)=\mathbf{R}_{\mathrm{os}}(\omega) \mathbf{K}_{\mathrm{ss}}(\omega) \mathbf{T}_{\mathrm{s}} \mathbf{U}_{\mathrm{s}}(\omega)
$$

\subsection{Vehicle, railway track and wheel-rail interaction}

The system is excited by one or more vehicles travelling across a railway track. The vehicles can be modelled using various multibody systems, with varying complexity, depending in the application case. Vehicles are only modelled in two dimensions and only the vertical wheel-rail interaction forces are considered. The dynamic stiffness matrix of the vehicle $\mathbf{K}_{\mathrm{vv}, i}\left(\omega_{\mathrm{m}}\right)$ is created in a moving FOR by combining the vehicle stiffness, damping and mass matrices. If multiple vehicles are needed, it is assumed that there is no direct interaction between vehicles and the dynamic stiffness matrix becomes:

$$
\mathbf{K}_{\mathrm{v}}\left(\omega_{\mathrm{m}}\right)=\left[\begin{array}{cccc}
\mathbf{K}_{\mathrm{vv}, 1}\left(\omega_{\mathrm{m}}\right) & \mathbf{0} & \cdots & \mathbf{0} \\
\mathbf{0} & \mathbf{K}_{\mathrm{vv}, 2}\left(\omega_{\mathrm{m}}\right) & \cdots & \mathbf{0} \\
\vdots & \vdots & \ddots & \vdots \\
\mathbf{0} & \mathbf{0} & \cdots & \mathbf{K}_{\mathrm{vv}, I}\left(\omega_{\mathrm{m}}\right)
\end{array}\right] .
$$


Here it is assumed that there is a total number $I$ of vehicles in the system. Further, there is no coupling between separate vehicles through the vehicle stiffness matrix, i.e. each car of a train acts like a separate vehicle.

A layered track structure containing the rails, rail-pads, sleepers and ballast is used. It is coupled to the underlying soil in the frequency-wavenumber domain as described in [15]. By using the stiffness matrix for the rails $\mathbf{K}_{\mathrm{r}}\left(\omega_{\mathrm{m}}\right)$ and the diagonal stiffness matrix $\mathbf{K}_{\mathrm{H}}$ for the wheel-rail contact interaction the coupled vehicle-track system response from a unit amplitude unevenness is given as:

$$
\left[\begin{array}{ccc}
\mathbf{K}_{\mathrm{v}}^{\mathrm{uu}}\left(\omega_{\mathrm{m}}\right) & \mathbf{K}_{\mathrm{v}}^{\mathrm{uw}}\left(\omega_{\mathrm{m}}\right) & \mathbf{0} \\
\mathbf{K}_{\mathrm{v}}^{\mathrm{wu}}\left(\omega_{\mathrm{m}}\right) & \mathbf{K}_{\mathrm{v}}^{\mathrm{ww}}\left(\omega_{\mathrm{m}}\right)+\mathbf{K}_{\mathrm{H}} & -\mathbf{K}_{\mathrm{H}} \\
\mathbf{0} & -\mathbf{K}_{\mathrm{H}} & \mathbf{K}_{\mathrm{r}}\left(\omega_{\mathrm{m}}\right)+\mathbf{K}_{\mathrm{H}}
\end{array}\right]\left\{\begin{array}{c}
\mathbf{U}_{\mathrm{v}}^{\mathrm{u}}\left(\omega_{\mathrm{m}}\right) \\
\mathbf{U}_{\mathrm{v}}^{\mathrm{w}}\left(\omega_{\mathrm{m}}\right) \\
\mathbf{U}_{\mathrm{r}}\left(\omega_{\mathrm{m}}\right)
\end{array}\right\}=\left\{\begin{array}{c}
\mathbf{0} \\
K_{\mathrm{H}} \mathbf{d}\left(\omega_{\mathrm{m}}\right) \\
-K_{\mathrm{H}} \mathbf{d}\left(\omega_{\mathrm{m}}\right)
\end{array}\right\}
$$

where $\mathbf{d}\left(\omega_{\mathrm{m}}\right)=\exp \left(\frac{\omega_{\mathrm{m}}}{v} \mathbf{x}_{\mathrm{w}}\right)$ is the vector containing the unit unevenness for all wheel positions $\mathbf{x}_{\mathrm{w}}$ in the moving FOR. In Eq. (19), the vehicle degrees of freedom are split into two parts: those relating to the wheels, denoted with the superscript ' $w$ ', and those that are not coupled to the track, denoted with superscript ' $u$ '. It is assumed that each vehicle wheel has the same linearized Hertzian spring stiffness $K_{\mathrm{H}}$ when calculating the acting forces. Solving Eq. (19) for the unknown displacement vector $\mathbf{U}$ produces the system behaviour. Note that in this case, the effects of external structures coupled to the railway track through the soil are not accounted for.

The stiffness matrix for the rails in Eq. (19) can be found by inverting the flexibility matrix $\mathbf{R}_{\mathrm{rr}}\left(\omega_{\mathrm{m}}\right)$ relating the rail displacements between all vehicle wheel sets positions is constructed. This is achieved in a similar manner as described in the previous section, assuming a single degree of freedom of a rail in a moving FOR for every wheel. The matrix is later used to establish the stiffness matrix of a coupled-domain stiffness matrix.

\section{ASSEMBLY AND SOLUTION OF GLOBAL SYSTEM}

Parts of the model described in the previous sections are combined into a single global system. The resulting system combines parts formulated in a moving FOR, such as the vehicle and the track, and parts formulated in the fixed FOR, such as the structures interacting with the soil. To couple the two FORs together, the relation described in Section 2 is used. For this purpose, the receptance matrix $\widetilde{\mathbf{R}}_{\mathrm{sr}}$, providing the interaction between the degrees of freedom of the rails and the degrees of freedom of the soil, is established. The matrix $\widetilde{\mathbf{R}}_{\mathrm{sr}}$ couples the moving and fixed FORs, and it is therefore dependent on two frequencies: $\omega_{\mathrm{m}}$ and $\omega_{\mathrm{f}}$. The tilde indicates that the quantity is defined in the mixed FOR.

\subsection{Partly coupled global system}

The flexibility matrix, assembled in the fixed FOR and connecting all the degrees of freedom interacting with the soil, is added to the system. Using the created matrices, the full flexibility matrix for the so-called 'global' (indicated by subscript 'g') system can be constructed

$$
\widetilde{\mathbf{R}}_{\mathrm{g}}\left(\omega_{\mathrm{m}}, \omega_{\mathrm{f}}\right)=\left[\begin{array}{cc}
\mathbf{R}_{\mathrm{rr}}\left(\omega_{\mathrm{m}}\right) & \widetilde{\mathbf{R}}_{\mathrm{rs}}\left(\omega_{\mathrm{f}}, \omega_{\mathrm{m}}\right) \\
\widetilde{\mathbf{R}}_{\mathrm{sr}}\left(\omega_{\mathrm{m}}, \omega_{\mathrm{f}}\right) & \mathbf{R}_{\mathrm{ss}}\left(\omega_{\mathrm{f}}\right)
\end{array}\right] .
$$

If no rigid bodies are present in the soil model, the global flexibility matrix may be inverted to obtain the stiffness matrix of the global system $\widetilde{\mathbf{K}}_{\mathrm{g}}\left(\omega_{\mathrm{m}}, \omega_{\mathrm{f}}\right)$. When the system contains rigid objects, which are formulated as described in Section 3.2, the global stiffness matrix can be constructed using the global transformation matrix 


$$
\mathbf{T}_{\mathrm{g}}=\left[\begin{array}{cc}
\mathbf{I} & \mathbf{0} \\
\mathbf{0} & \mathbf{T}_{\mathrm{s}}
\end{array}\right]
$$

where $\mathbf{I}$ is the identity matrix with dimensions equal to the number of rail degrees of freedom. It is assumed that there are no rigid bodies modelled in the moving FOR. In theory, it is possible to model rigid bodies in both the moving and the fixed FORs, given that the reference nodes for these bodies are not shared between the two FORs.

After the stiffness matrix of the system has been obtained, the vehicle and the FE structures can be also added to the global system. The governing equation for partial coupling in the mixed FOR becomes:

$$
\widetilde{\mathbf{K}}\left(\omega_{\mathrm{m}}, \omega_{\mathrm{f}}\right) \widetilde{\mathbf{U}}\left(\omega_{\mathrm{m}}, \omega_{\mathrm{f}}\right)=\tilde{\mathbf{F}}\left(\omega_{\mathrm{m}}, \omega_{\mathrm{f}}\right)
$$

where

$$
\widetilde{\mathbf{K}}\left(\omega_{\mathrm{m}}, \omega_{\mathrm{f}}\right)=\left[\begin{array}{ccccc}
\mathbf{K}_{\mathrm{v}}^{\mathrm{uu}}\left(\omega_{\mathrm{m}}\right) & \mathbf{K}_{\mathrm{v}}^{\mathrm{uw}}\left(\omega_{\mathrm{m}}\right) & \mathbf{0} & \mathbf{0} & \mathbf{0} \\
\mathbf{K}_{\mathrm{v}}^{\mathrm{wu}}\left(\omega_{\mathrm{m}}\right) \mathbf{K}_{\mathrm{v}}^{\mathrm{ww}}\left(\omega_{\mathrm{m}}\right)+\mathbf{K}_{\mathrm{H}} & -\mathbf{K}_{\mathrm{H}} & \mathbf{0} & \mathbf{0} \\
\mathbf{0} & -\mathbf{K}_{\mathrm{H}} & \widetilde{\mathbf{K}}_{\mathrm{g}}^{\mathrm{rr}}\left(\omega_{\mathrm{m}}, \omega_{\mathrm{f}}\right)+\mathbf{K}_{\mathrm{H}} & \widetilde{\mathbf{K}}_{\mathrm{g}}^{\mathrm{rs}}\left(\omega_{\mathrm{f}}, \omega_{\mathrm{m}}\right) & \mathbf{0} \\
\mathbf{0} & \mathbf{0} & \widetilde{\mathbf{K}}_{\mathrm{g}}^{\mathrm{sr}}\left(\omega_{\mathrm{m}}, \omega_{\mathrm{f}}\right) & \widetilde{\mathbf{K}}_{\mathrm{g}}^{\mathrm{ss}}\left(\omega_{\mathrm{m}}, \omega_{\mathrm{f}}\right)+\widetilde{\mathbf{K}}_{\mathrm{FE}}^{\mathrm{ss}}\left(\omega_{\mathrm{f}}\right) & \mathbf{K}_{\mathrm{FE}}^{\mathrm{sn}}\left(\omega_{\mathrm{f}}\right) \\
\mathbf{0} & \mathbf{0} & \mathbf{0} & \mathbf{K}_{\mathrm{FE}}^{\mathrm{ns}}\left(\omega_{\mathrm{f}}\right) & \mathbf{K}_{\mathrm{FE}}^{\mathrm{nn}}\left(\omega_{\mathrm{f}}\right)
\end{array}\right]
$$

and

$$
\widetilde{\mathbf{U}}\left(\omega_{\mathrm{m}}, \omega_{\mathrm{f}}\right)=\left\{\begin{array}{c}
\widetilde{\mathbf{U}}_{\mathrm{v}}^{\mathrm{u}}\left(\omega_{\mathrm{m}}, \omega_{\mathrm{f}}\right) \\
\widetilde{\mathbf{U}}_{\mathrm{v}}^{\mathrm{w}}\left(\omega_{\mathrm{m}}, \omega_{\mathrm{f}}\right) \\
\widetilde{\mathbf{U}}_{\mathrm{g}}^{\mathrm{r}}\left(\omega_{\mathrm{m}}, \omega_{\mathrm{f}}\right) \\
\widetilde{\mathbf{U}}_{\mathrm{g}}^{\mathrm{s}}\left(\omega_{\mathrm{m}}, \omega_{\mathrm{f}}\right) \\
\widetilde{\mathbf{U}}_{\mathrm{FE}}^{\mathrm{n}}\left(\omega_{\mathrm{m}}, \omega_{\mathrm{f}}\right)
\end{array}\right\}, \quad \tilde{\mathbf{F}}\left(\omega_{\mathrm{m}}, \omega_{\mathrm{f}}\right)=\left\{\begin{array}{c}
\mathbf{0} \\
K_{\mathrm{H}} \mathbf{d}\left(\omega_{\mathrm{m}}\right) \\
-K_{\mathrm{H}} \mathbf{d}\left(\omega_{\mathrm{m}}\right) \\
\mathbf{0} \\
\mathbf{0}
\end{array}\right\} .
$$

The superscripts relate to the degrees of freedom of: ' $w$ '-wheels, ' $r$ '-rails, 's'-soil in the fixed FOR, ' $n$ '-parts of FE structures not coupled to the soil and 'u'-vehicles uncoupled from the track (e.g. the vehicle body). Vectors $\widetilde{\mathbf{U}}_{\mathrm{v}}, \widetilde{\mathbf{U}}_{\mathrm{g}}$ and $\widetilde{\mathbf{U}}_{\mathrm{FE}}$ store the displacements for the vehicle, global railway track-soil system and the FE structure, respectively.

After the system has been solved, the displacement for the observation degrees of freedom in the fixed FOR can be obtained. When the displacements for both the global and the observation degrees of freedom have been obtained, the effects from $J_{\mathrm{m}}$ discrete excitation frequencies $\omega_{\mathrm{m}}$ can be added together to obtain the total response in the fixed FOR:

$$
\mathbf{U}_{\mathrm{FE}}\left(\omega_{\mathrm{f}}\right)=\frac{1}{2 \pi} \sum_{j=1}^{J_{\mathrm{m}}} \widetilde{\mathbf{U}}_{\mathrm{FE}}\left(\omega_{\mathrm{m}, j}, \omega_{\mathrm{f}}\right) D\left(k_{\mathrm{m}, j}\right) \Delta k_{\mathrm{m}}
$$

where $D\left(k_{\mathrm{m}, j}\right)$ is the rail unevenness obtained from a power spectral density (PSD) according to the wavenumber $k_{\mathrm{m}, j}$, and $\Delta k_{\mathrm{m}}$ is the wavenumber step size.

The time-domain response for the degrees of freedom associated with the fixed FOR can be obtained by performing an inverse discrete Fourier transformation of the displacements $\mathbf{U}_{\mathrm{FE}}\left(\omega_{\mathrm{s}}\right)$ :

$$
\mathbf{u}_{\mathrm{FE}}(t)=\frac{1}{2 \pi} \sum_{j=1}^{J_{\mathrm{f}}} \mathbf{U}_{\mathrm{FE}}\left(\omega_{\mathrm{f}, j}\right) \mathrm{e}^{\mathrm{i} \omega_{\mathrm{f}, j} t} \Delta \omega_{\mathrm{f}}
$$

where $J_{\mathrm{f}}$ is the number of discrete frequencies in the fixed FOR. Note that $J_{\mathrm{f}}$ and $J_{\mathrm{m}}$ need not be equal. However, the step sizes $\Delta \omega_{\mathrm{f}}$ and $\Delta k_{\mathrm{m}}$ must be small enough, and the number of frequencies $J_{\mathrm{f}}$ and $J_{\mathrm{m}}$ large enough, to ensure proper discretization of peaks in the loads and resonances 
of the system while, at the same time, avoiding violation of the periodicity inherent in the frequency-domain solution.

\subsection{Fully coupled global system}

Using the matrices already created for the previously described solution procedure, it is also possible to assemble a fully coupled global system. Such a system will account for the frequency spreading of the reflected waves due to the Doppler effect and might be useful for certain cases where the full coupling between the vehicle (the source) and the structures (the receivers) cannot be discarded. In such a system, the previously uncoupled frequencies in a single FOR are now coupled through the other FOR and the matrix $\mathbf{K}_{\mathrm{g}}$ will be fully populated. The system can be solved by applying loads in either FOR or both FORs at the same time. However, the computation of the fully coupled system is an extremely computationally demanding process, which involves double inversion of very large matrices. Thus, it should only be used when the secondary coupling effects are an important factor.

\section{VALIDATION AND APPLICATIONS}

The methodology described in this paper is validated by comparisons with other state-ofthe-art computational approaches. By considering a system with no structures or rigid objects interacting with the soil, the described system assembly and solution procedures provide identical results to the approach provided by Sheng et al. in $[13,14]$. This is expected, as the proposed method here utilizes the same vehicle-track-soil interaction model, and, with no other structures interacting with the soil, the coupling terms cancel out. Modelling of rigid objects interacting with the soil was validated by comparison with BE and FEM-PML models. It was determined that the semi-analytical model provides a very good match, especially with the FEM-PML model, where even the secondary coupling terms show very good agreement. To demonstrate the capabilities of the proposed fully-coupled modelling approach, an example case was set up where the modelled structure is close to the railway track.

\subsection{Coupling between moving and fixed frames of reference}

In order to validate the coupling terms between the two FORs and especially the symmetry between when a load is applied in the moving FOR and the displacements observed in a fixed FOR and vice versa, two test cases were set up. The proposed mixed-FOR model was simplified by removing the railway track, the vehicle and the FE/rigid structures interacting with the soil. This way, the effects of a single load with a single excitation frequency acting directly on the soil surface can be observed. The ground was modelled as a homogenous elastic half-space of dense sandy-type soil, with $250 \mathrm{MPa}$ Young's modulus, 0.25 Poisson's ratio, $2000 \mathrm{~kg} / \mathrm{m}^{3}$ mass density and 0.05 loss factor. A stationary point in the fixed FOR was placed $3 \mathrm{~m}$ from the line along which the load was moving.

A moving vertical load with constant speed $v$ and frequency $f_{\mathrm{m}}$ was modelled. The vertical displacements were observed within a fixed FOR for a range of frequencies $f_{\mathrm{f}}$. The system was modelled using the simplified mixed-FOR model. For the analysed case, only the coupling terms between the moving and the fixed FORs, as described in Section 2, have an effect for the obtained results. For comparison, a full model was established, modelled only in the moving FOR, using the semi-analytical approach.

Using the created model, the displacement field of the soil surface in the moving FOR and within the time domain was obtained for a single excitation frequency. Then, a time signal for displacements of an observation point moving through the displacement field with speed $-v$ was found, considering that for every time step the position of the observation point changed. 
Fourier transforming the obtained time signal into frequencies $f_{\mathrm{s}}$, the displacement spectra for a stationary observation point was obtained. Two combinations of speed and frequency in the moving FOR were investigated: Case 1 with $v=20 \mathrm{~m} / \mathrm{s}$ and $f_{\mathrm{m}}=20 \mathrm{~Hz}$, and Case 2 with $v=$ $40 \mathrm{~m} / \mathrm{s}$ and $f_{\mathrm{m}}=10 \mathrm{~Hz}$. The comparison of both approaches are shown in Figure 1 where it can be seen that the two approaches agree, confirming the analytical derivation presented in Section 2.

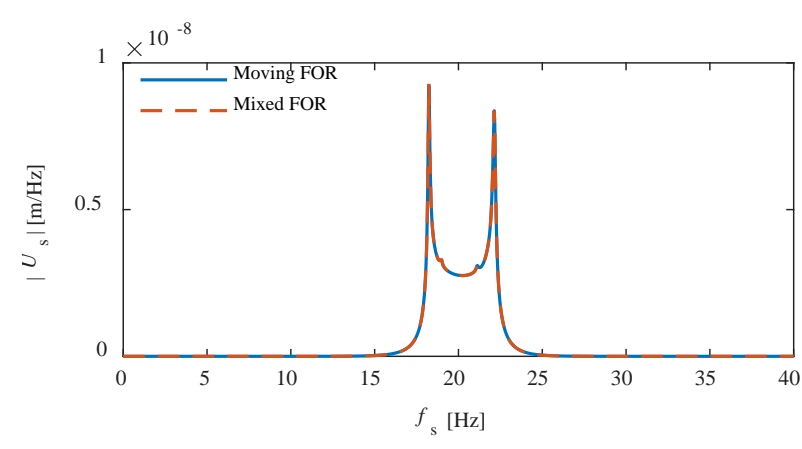

(a)

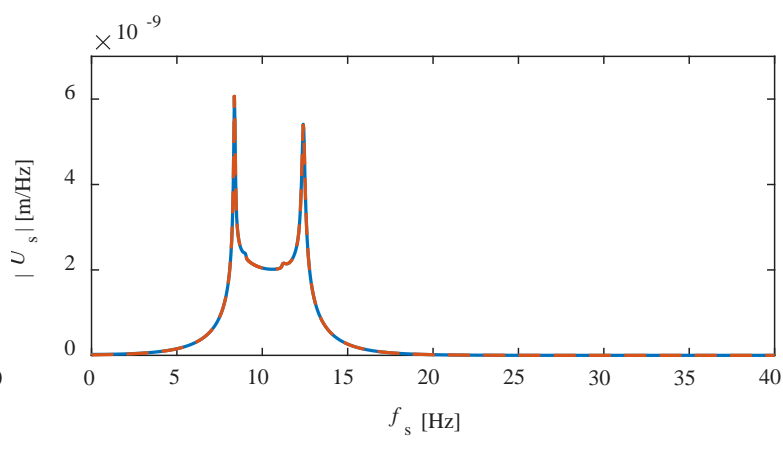

(b)

Figure 1: Displacements in a fixed FOR from a unit load applied in a moving FOR: (a) $v=20 \mathrm{~m} / \mathrm{s}$ and $f_{\mathrm{m}}=$ $20 \mathrm{~Hz}$; (b) $v=40 \mathrm{~m} / \mathrm{s}$ and $f_{\mathrm{m}}=10 \mathrm{~Hz}$.

A similar validation example was created to analyse the coupling between a load in a fixed FOR and the resulting displacements in a moving FOR. Here, the mixed FOR model was compared to a full solution formulated only in a fixed FOR. In the same way as for the previous validation case, using the full model, the displacement field of the soil surface was found in time domain. Then the vertical displacements for a moving observation point were obtained by changing the position of the point for every time step. Fourier transforming the time-domain response for frequencies $f_{\mathrm{m}}$, the displacement spectra for a moving observation point were obtained. Two combinations of speed and stationary frequency were again investigated: Case 1 with $v=20 \mathrm{~m} / \mathrm{s}$ and $f_{\mathrm{s}}=20 \mathrm{~Hz}$; Case 2 with $v=40 \mathrm{~m} / \mathrm{s}$ and $f_{\mathrm{s}}=10 \mathrm{~Hz}$. Results of both approaches are given in Figure 2. Once again, the results agree well, this time confirming the derivation given in Section 2.

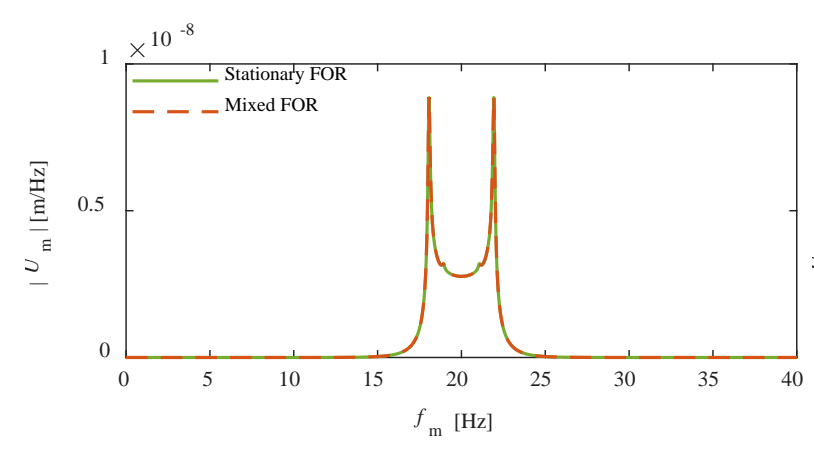

(a)

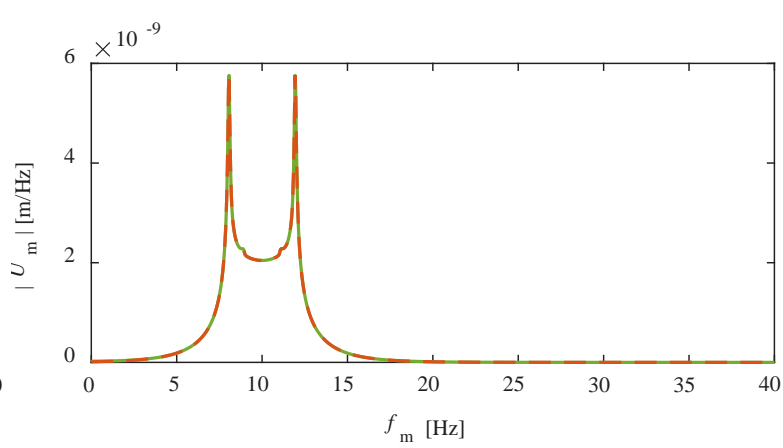

(b)

Figure 2: Displacements in a moving FOR from a unit load applied in a fixed FOR.: (a) $v=20 \mathrm{~m} / \mathrm{s}$ and $f_{\mathrm{s}}=$ $20 \mathrm{~Hz}$; (b) $v=40 \mathrm{~m} / \mathrm{s}$ and $f_{\mathrm{s}}=10 \mathrm{~Hz}$.

Comparing the two validation examples, it is evident that the response spreads out through the observer frequencies due to the Doppler effect, independently of which FOR the load was 
applied in. The range of affected frequencies dependent on the speed of the moving FOR. However, a load applied in the fixed FOR produces two symmetric peaks around the excitation frequency, when observed in a moving FOR, while a load applied in a moving FOR produces two peaks in the fixed FOR. Further, comparing the results of Case 1 for both cases, it can be observed that the result in Figure 4 at $f_{\mathrm{s}}=20 \mathrm{~Hz}$ and the result in Figure 5 at $f_{\mathrm{m}}=20 \mathrm{~Hz}$ show exactly the same response. This confirms the symmetry between the two FORs implied by Eq. (7). The same result is obtained by comparing the responses of Case 2 for both cases.

\subsection{Validation of the modelling approach}

To validate the modelling approach presented in this paper, it was compared to a sub-modelling technique, as described in [6]. A similar modelling approach was also used in [5]. In both cases the sub-modelling approach was used to model an underground railway tunnel with a vehicle travelling through it, in turn exciting a building structure above the soil. However, the solution procedure can also be applied to surface railways and the system analysed in this work. The method uses the free-field displacements of the soil caused by a passing vehicle, which are later modified by introducing the building structure. Ensuring equilibrium and compatibility between the degrees of freedom connecting the building and the soil, a solution for the building displacements can be found.

Comparing the methodology proposed in this work with the sub-modelling technique, it becomes evident that the basic parts used for both solution procedures are identical. For example, the free-field displacements from a moving load used in the two-step approach are identical to the flexibility matrix used here. However, the assembly and solution of the full system is somewhat different, with the proposed methodology allowing a wider range of applications, such as modelling rigid inclusions or allowing a two-way coupling between the two FORs. Further, the sub-modelling technique, as applied in this work, is a two-step solution approach, as the wheelrail interaction forces are obtained in the moving FOR before being used to obtain the displacements in the fixed FOR.

As, both approaches use the same basic parts, it is relatively easy to compare them. For comparison, a building structure, with six columns supporting two floors was used. The building is facing the railway track with its narrow side, which is $8 \mathrm{~m}$ wide, and the building is supported by two columns at either side. The length of the building is $10 \mathrm{~m}$ in the direction orthogonal to the track. In addition to the four columns placed at the corners, a column is placed in the middle of each of the longer sides. Each storey is $4 \mathrm{~m}$ high. The whole building structure is constructed from concreate with a Young's modulus of $30 \mathrm{GPa}$, a Poisson's ratio of 0.15 , a mass density of $2400 \mathrm{~kg} / \mathrm{m} 3$, and loss factor of 0.03 . The columns have square cross-sections, with one side equal to $0.3 \mathrm{~m}$, while the floors are $0.25 \mathrm{~m}$ thick slabs. Since the sub-modelling technique cannot directly model rigid objects, a flexible slab footing lies underneath the whole building with the same thickness as the building floors. 3D beam elements were used to model the columns, using Euler-Bernoulli beam theory to account for bending. The floors and the foundation slab of the building were modelled using Mindlin-Reissner shell elements, accounting for bending as well as shear. All FE parts of the model were discretized with mesh size of $0.5 \mathrm{~m}$, resulting in 4841 degrees of freedom in the system.

The soil is modelled as a half-space of sand, with the same properties as in Section 5.1. Both systems were excited by a single passing vehicle, travelling at $40 \mathrm{~m} / \mathrm{s}$, exposed to a unit rail unevenness, with a $4 \mathrm{~m}$ wavelength corresponding to an excitation frequency of $10 \mathrm{~Hz}$. For analysis, only a single excitation frequency is used, as the effects from multiple excitations 
frequencies are just added together due to the principal of superposition. Thus, a single excitation frequency is enough to evaluate how well both models perform. The test case is illustrated in Figure 3.

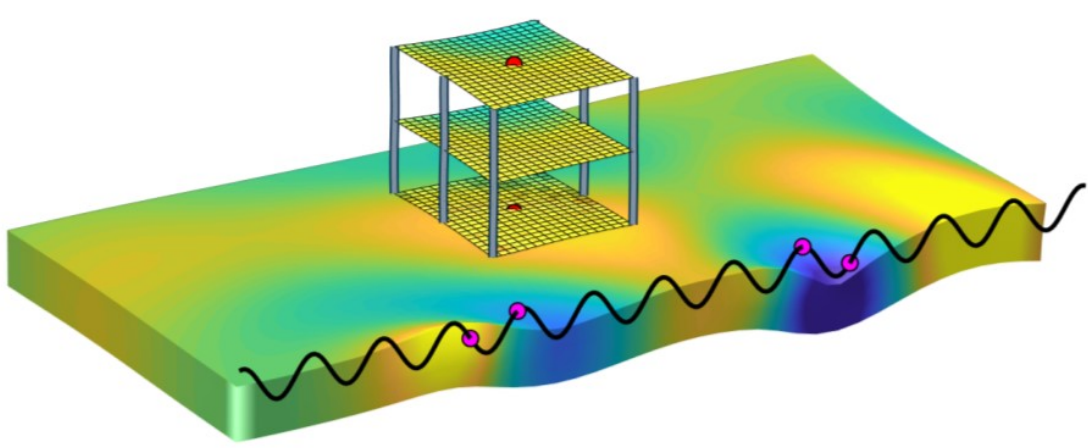

Figure 3: Investigated case for validating the modelling approach. The vehicle is travelling from left to right, with magenta nodes indicating the wheel positions. The red nodes indicate the observation points, while the shades of colour indicate the vertical displacements (bright yellow is up, dark blue is down). Track unevenness is not scaled.

Figure 4 shows the displacements of the building structure obtained at the centre of the ground floor and the second floor where both approaches provide almost identical results, showing that the proposed method is performing well. At the same time, almost identical results indicate that the back-coupling of the building structure to the railway track is insignificant. Thus, for at least for the considered case, there is no significant difference whether on or the other approach is considered.

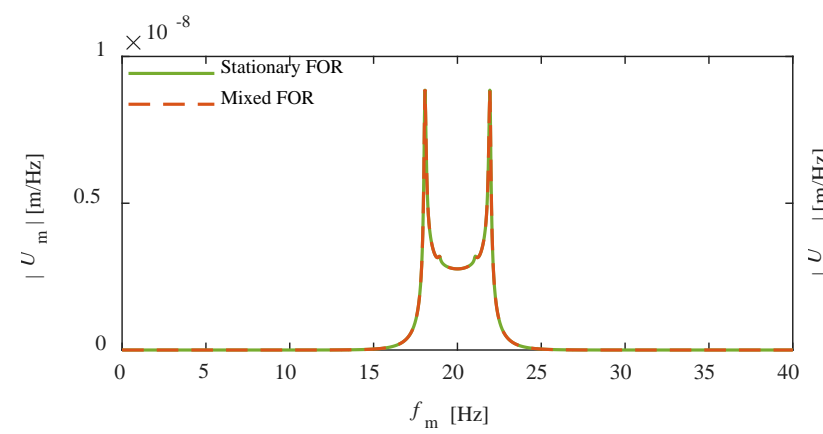

(a)

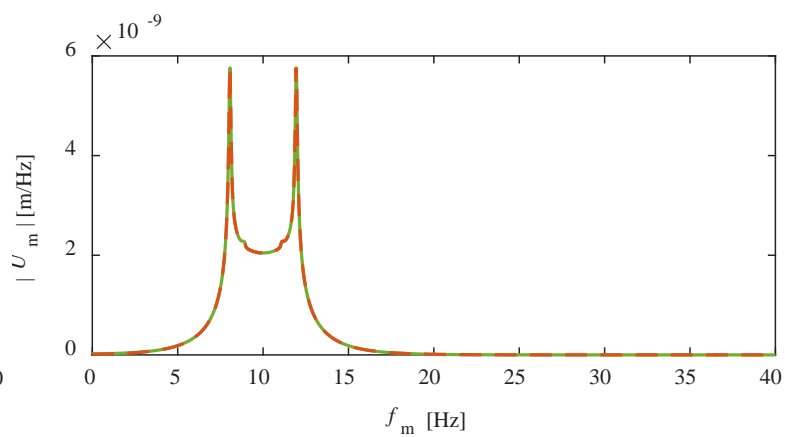

(b)

Figure 4: Displacements in a moving FOR from a unit load applied in a fixed FOR.: (a) $v=20 \mathrm{~m} / \mathrm{s}$ and $f_{\mathrm{s}}=$ $20 \mathrm{~Hz}$; (b) $v=40 \mathrm{~m} / \mathrm{s}$ and $f_{\mathrm{s}}=10 \mathrm{~Hz}$.

\subsection{Fully coupled system}

A fully-coupled system solution approach might be necessary in cases where the modelled structures are close to the railway track, introducing a significant change of dynamic stiffness along the track. In that case, the re-scattered waves can have an effect on the vehicle behaviour and the obtained wheel-rail interaction forces. Problems where these effects are important could include modelling of tunnels underneath buildings, railway stations and various structures nearby the tracks. Several such cases have been investigated by Coulier et al. [20], finding that while the axial loads are not effected significantly, the vibration insertion gain for source-receiver transmission can be affected up to $10 \mathrm{~dB}$, when using a fully coupled solution procedure. 
Another set of problems could include the sudden change of stiffness underneath the track, e.g. when a concreate passage is constructed underneath the railway track.

To examine such a case, a test case was set up modelling a single vehicle traveling across a railway track, as shown in Figure 5. The speed of the vehicle was $40 \mathrm{~m} / \mathrm{s}$. The vehicle and the track properties are given in Tables 1 and 2. The soil was modelled as a $5 \mathrm{~m}$ layer of soft clay sitting over a stiffer half-space of sand. The clay had a Young's modulus of $80 \mathrm{MPa}$, a Poisson's ratio of 0.48 , a mass density of $2100 \mathrm{~kg} / \mathrm{m} 3$, and a loss factor of 0.05 . The underlying sand had the same properties as in Section 5. Underneath the track, at a depth of $1 \mathrm{~m}$, a rigid block was embedded within the soil. The block was centred at the position, where the travelling vehicle centre line was located at time 0 . The block was modelled as a $2 \mathrm{D}$ plate, placed in the horizontal plane with one side equal to $2 \mathrm{~m}$. It was discretized into 36 discretization nodes, with three degrees of freedom per node. The system was excited by the deadweight of the vehicle only applied at $f_{\mathrm{m}}=0 \mathrm{~Hz}$, with no excitation from the rail unevenness. The system was assessed by the fully-coupled modelling approach. As only the quasi-static effects of the vehicle were modelled, the considered frequency ranges are reduced. The one-sided frequency range of the moving FOR was $0-30 \mathrm{~Hz}$, and the one-sided frequency range of the fixed FOR is $0-25 \mathrm{~Hz}$. In the computation, negative as well as positive frequencies were considered for either FOR, and each range was split into 200 discrete frequencies.

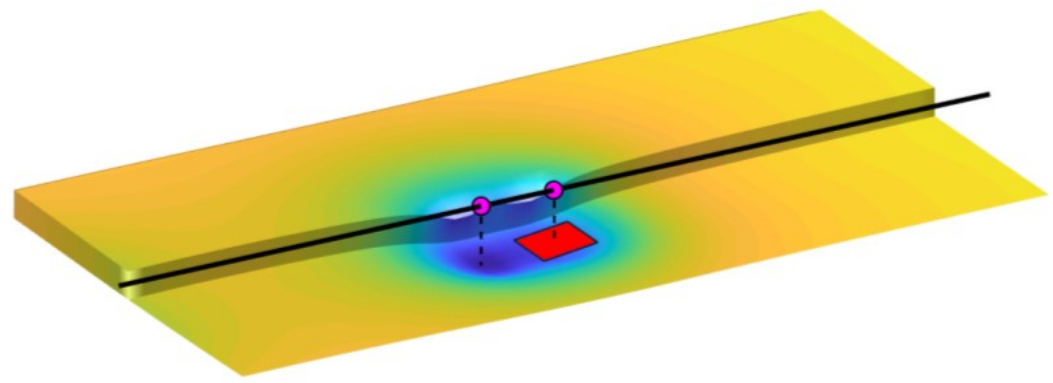

Figure 5: Vehicle passing over a buried rigid block, at an instance where the leading wheel of the vehicle is directly above the centre of the rigid block. The vehicle is travelling at $40 \mathrm{~m} / \mathrm{s}$ from left to right. The black line indicates the vehicle traverse line, with the magenta nodes indicating the positions of the vehicle wheel sets. Only the two wheel sets of the front bogie are shown.

\begin{tabular}{ll}
\hline Rail mass per unit length & $60.0 \mathrm{~kg} / \mathrm{m}$ \\
Rail bending stiffness & $6.4 \cdot 10^{6} \mathrm{~N} / \mathrm{m}^{2}$ \\
Rail loss factor & 0.01 \\
Railpad stiffness & $5.0 \cdot 10^{8} \mathrm{~N} / \mathrm{m}$ \\
Railpad loss factor & 0.1 \\
Sleeper spacing & $1 \mathrm{~m}$ \\
Sleeper mass per unit length & $542.0 \mathrm{~kg} / \mathrm{m}$ \\
Ballast vertical stiffness & $4.64 \cdot 10^{9} \mathrm{~N} / \mathrm{m}^{2}$ \\
Ballast mass per unit length & $1740 \mathrm{~kg} / \mathrm{m}$ \\
Ballast loss factor & 0.04 \\
Track width & $3.2 \mathrm{~m}$ \\
\hline
\end{tabular}

Table 1: Railway track properties.

Figure 6 shows the velocities obtained for the vehicle in the moving FOR as well as the rigid block in the fixed FOR. It can be observed that the vehicle passing over a rigid block introduces a significant excitation into the system, with the rigid block as well as the vehicle reacting to the passage. In this system, the observed excitation of the vehicle is purely due to the weak 
coupling effects that are disregarded in the two-step procedure. The waves generated by the passing vehicle are scattered by the rigid block in the fixed FOR and in turn excite a range of frequencies in the moving FOR. These effects would not be accounted for when the weak coupling between the vehicle and structures is not modelled.

\begin{tabular}{ll}
\hline Mass of car body & $40000 \mathrm{~kg}$ \\
Mass of bogie & $5000 \mathrm{~kg}$ \\
Mass of wheel set & $1800 \mathrm{~kg}$ \\
Car body pitch moment of inertia & $2.0 \cdot 10^{6} \mathrm{~kg} \cdot \mathrm{m}^{2}$ \\
Bogie pitch moment of inertia & $6000 \mathrm{~kg} \cdot \mathrm{m}^{2}$ \\
Primary suspension stiffness & $2.4 \cdot 10^{6} \mathrm{~N} / \mathrm{m}$ \\
Secondary suspension stiffness & $6.0 \cdot 10^{5} \mathrm{~N} / \mathrm{m}$ \\
Primary suspension damping & $30 \cdot 10^{3} \mathrm{~N} \cdot \mathrm{s} / \mathrm{m}$ \\
Secondary suspension damping & $20 \cdot 10^{3} \mathrm{~N} \cdot \mathrm{s} / \mathrm{m}$ \\
Distance between bogies' centers & $19.0 \mathrm{~m}$ \\
Distance between bogie's wheels sets & $2.7 \mathrm{~m}$ \\
Herztian constant & $5.14 \cdot 10^{-8}$ \\
\hline
\end{tabular}

Table 2: Vehicle properties.



(a)

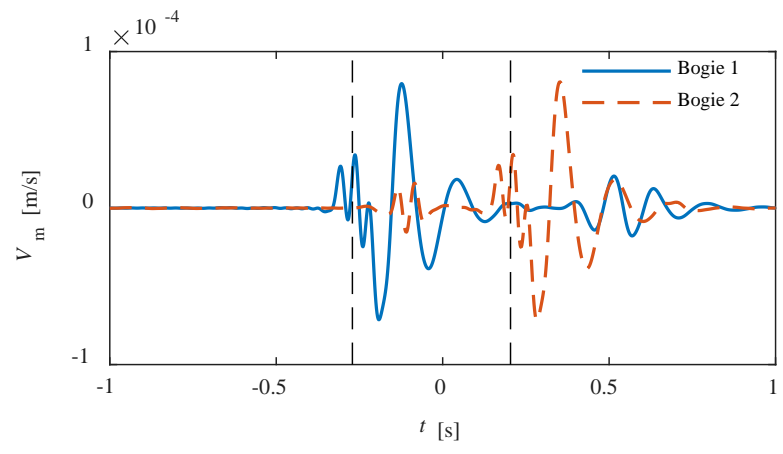

(b)

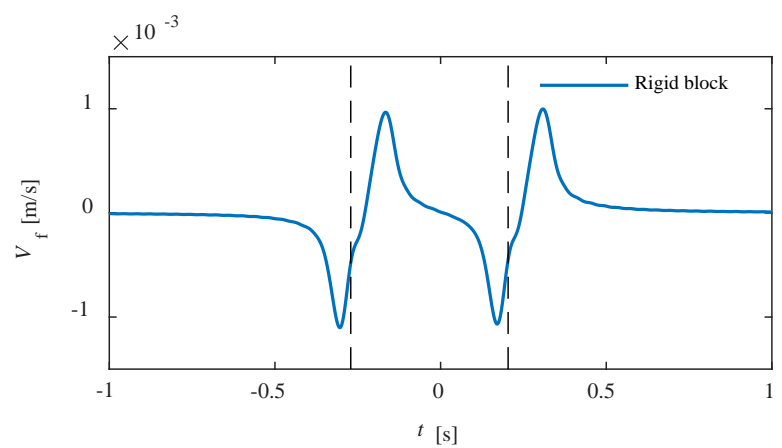

(c)

Figure 6: Vertical velocities for parts of the vehicle (in moving FOR) and the rigid block underneath the track (in fixed FOR). The vertical dashed black lines indicate time instance when the first and third vehicle wheels are directly above the centre of the rigid block. The response is shown for: (a) the first and the third vehicle wheels;

(b) both vehicle bogies; (c) rigid block underneath the track.

From the wheel velocities shown in Figure 6(a), it can be seen that both leading wheels of separate bogies produce very similar results. However, the excitation due to the first wheel passage over the rigid block generates a wave that excites the third vehicle wheel, even before 
it reaches the rigid block. A similar effect is also observed after the third wheel passes over the rigid block, where the generated wave travels forward and excites the first vehicle wheel. A very similar behaviour is also observed on the bogies. When comparing these secondary excitations, it can be seen that the reaction of the third wheel due to the first wheel passage is higher than the excitation of the first wheel due to third wheel passage. This is due to the Doppler effect of the wave travelling through the rail, as the third wheel is travelling towards the wave propagating form the first wheel, while the wave generated by the third wheel must 'catch up' to the first wheel. From the rigid block displacements, it can be observed that the largest velocities are reached just before the leading wheel of a bogie reaches the centre of block. Interestingly, to obtain the same displacements of the rigid block, a fully-coupled system solution approach is unnecessary, as the partly coupled solution provides results that are almost identical. For the analysed case, it can beconcluded that the fully coupled solution approach is only necessary if the vehicle behaviour is of interest, for example for assessment of driver and passenger comfort, or if the track and wheel wear is to be assessed.

\section{CONCLUSIONS}

- The paper introduced a new modelling approach for the estimation of environmental vibration resulting from railway traffic. A model of a coupled vehicle-track-soil-building system was introduced with two approaches proposed for assembly and solution of the whole system: the partly-coupled approach and the fully-coupled approach. Both solution approaches use a single step procedure with one approach considering a fully-coupled system, while the other approach discards some secondary coupling effects for a more computationally efficient solution procedure.

- A semi-analytical model was utilized to model the soil to which rigid objects and structures modelled by the FE were coupled. The proposed modelling approach uses the frequencydomain solution with some parts, such as the railway track, formulated in the moving FOR and other parts, such as building structures, formulated in a fixed FOR. The coupling terms between the two FORs are found by utilizing an analytical formulation of receptance between the two FORs. It has been established that due to the coupling between the fixed and moving FORs, the previously uncoupled discrete frequencies become coupled through the other FOR as a result of the Doppler effect and wave scattering. Thus, additional consideration is needed when modelling such systems.

- The proposed methodology is a robust approach that does not suffer from numerical instabilities, due to the usage of frequency domain solutions. A wide range of cases can be assessed, including the modelling of rigid objects in or on the ground and flexible structures modelled by the FEM, interacting with the soil. Using the proposed partly-coupled solution approach, the computations can be easily parallelized, thus providing a relatively fast and efficient computational method. Further, the suggested fully-coupled solution procedure is useful in cases where the weak coupling between the vehicle and structures cannot be discarded.

- The analytically derived coupling terms between the two FORs were validated by comparing with models formulated in a single FOR. Further, the partly coupled solution procedure of the full system was compared to a solution procedure in which the weak coupling is completely discarded. The two solution procedures have been found to provide almost identical results, validating the proposed partly coupled solution procedure while, at the same time, indicating insignificant back-coupling in the considered case. 
- To demonstrate the capabilities of the numerical model and the potential of the fully-coupled solution procedure, the response of a vehicle passing over a buried rigid block was investigated. It was been found that the fully coupled solution procedure can predict the weak coupling effects between the vehicle and the structure, producing a vehicle response distributed through frequencies, when the load is applied only at a single frequency. Thus, in the present example, the modes of the vehicle were excited parametrically by the waves scattered from the rigid inclusion as a result of the passing deadweight of the vehicle.

\section{REFERENCES}

[1] D.J. Thompson, G. Kouroussis, E. Ntotsios, Modelling, simulation and evaluation of ground vibration caused by rail vehicles, Vehicle System Dynamics 57, 69-110, 2019.

[2] P. Fiala, G. Degrande, F. Augusztinovicz, Numerical modelling of ground-borne noise and vibration in buildings due to surface rail traffic, Journal of Sound and Vibration, 301, 718-738, 2007.

[3] S. François, L. Pyl, H.R. Masoumi, G. Degrande, The influence of dynamic soil-structure interaction on traffic induced vibrations in buildings, Soil Dynamics and Earthquake Engineering 27, 655-674, 2007.

[4] G. Kouroussis, L. Van Parys, C. Conti, O. Verlinden, Prediction of ground vibrations induced by urban railway traffic: an analysis of the coupling assumptions between vehicle, track, soil, and buildings, International Journal of Acoustics and Vibration 163, 163-172, 2013.

[5] P. Lopes, P.A. Costa, M. Ferraz, R. Calçada, A.S. Cardoso, Numerical modeling of vibrations induced by railway traffic in tunnels: From the source to the nearby buildings, Soil Dynamics and Earthquake Engineering 61-62, 269-285, 2014.

[6] M.F.M. Hussein, H. Hunt, K. Kuo, P.A. Costa, J. Barbosa, The use of sub-modelling technique to calculate vibration in buildings from underground railways, Proceedings of the Institution of Mechanical Engineers, Part F: Journal of Rail and Rapid Transit, 229, 303-314, 2015.

[7] D. López-Mendoza, A. Romero, D.P. Connolly, P. Galvín, Scoping assessment of building vibration induced by railway traffic, Soil Dynamics and Earthquake Engineering, 93, 147-161, 2017.

[8] D.P. Connolly, P. Galvín, B. Olivier, A. Romero, G. Kouroussis, A 2.5D time-frequency domain model for railway induced soil-building vibration due to railway defects, Soil Dynamics and Earthquake Engineering, 120, 332-344, 2019.

[9] K.A. Kuo, M. Papadopoulos, G. Lombaert, G. Degrande, The coupling loss of a building subject to railway induced vibrations: Numerical modelling and experimental measurements, Journal of Sound and Vibration, 442, 459-481, 2019.

[10] P. Coulier, G. Lombaert, G. Degrande, The influence of source-receiver interaction on the numerical prediction of railway induced vibrations, Journal of Sound and Vibration, 333(12), 2520-2538, 2014 
[11] M.F.M. Hussein, H.E.M. Hunt, A numerical model for calculating vibration from a railway tunnel embedded in a full-space, Journal of Sound and Vibration, 305, 401-431, 2007.

[12] C. Hanson, D. Towers, L. Meister, Transit Noise and Vibration Impact Assessment, Report FTA-VA-90-1003-06, U.S. Department of Transportation, Federal Transit Administration, Office of Planning and Environment, 2006.

[13] X. Sheng, C.J.C. Jones, M. Petyt, Ground vibration generated by a load moving along a railway track, Journal of Sound and Vibration, 228, 129-156, 1999.

[14] X. Sheng, C.J.C. Jones, D.J. Thompson, A theoretical model for ground vibration from trains generated by vertical track irregularities, Journal of Sound and Vibration, 272, 937965, 2004.

[15] E. Ntotsios, D.J. Thompson, M.F.M. Hussein, The effect of track load correlation on ground-borne vibration from railways, Journal of Sound and Vibration, 402, 142-163, 2017.

[16] W.T. Thomson, Transmission of elastic waves through a stratified solid medium, Journal of Applied Physics, 21, 89-93, 1950.

[17] N.A. Haskell, The dispersion of surface waves on multilayered media, Bulletin of the Seismological Society of America, 43, 17-43, 1953.

[18] E. Kausel, J.M. Roësset, Stiffness matrices for layered soils, Bulletin of the Seismological Society of America, 71, 1743-1761, 1981.

[19] R. Wang, A simple orthonormalization method for stable and efficient computation of Green's functions, Bulletin of the Seismological Society of America, 89, 733-741, 1999.

[20] P. Coulier, G. Lombaert, G. Degrande, The influence of source-receiver interaction on the numerical prediction of railway induced vibrations, Journal of Sound and Vibration, 333, 2520-2538, 2014. 